\title{
Nümerik Optimizasyon için Kaotik Altın Sinüs Algoritması
}

\author{
Erkan TANYILDIZI ${ }^{*}$, Gökhan DEMİR \\ ${ }^{1}$ Fırat Üniversitesi Teknoloji Fakültesi Yazılım Mühendisliği Bölümü, Elazığ \\ ${ }^{2}$ Fırat Üniversitesi Teknoloji Fakültesi Yazılım Mühendisliği Bölümü, Elazığ \\ *1 etanyildizi@ firat.edu.tr, ${ }^{2}$ gokhan.demir.dg@gmail.com
}

Özet: Rastgele sayı dizileri, sezgisel optimizasyon algoritmalarının stokastik yapılarından dolayı sıkça kullanılmaktadır. Sezgisel optimizasyon algoritmaların global optimuma yakınsamalarında rastgele üretilen sayı dizilerinin dağılımları oldukça etkili olmaktadır. Rastgele üretilen sayıların belli bir alanda toplanması veya aynı değerler üretilmesi ile algoritmanın lokal optimumlara takılma riski artabilmektedir. Bu nedenle üretilen sayıların aynı olmaması, yayılmış spektruma sahip olması sezgisel algoritmaların performansını etkileyen durumlardır. Kaotik haritalar ayrık zamanlı sistemlerdir ve kaotik haritalarla üretilen sayılar geniş bir spektruma sahip olup periyodik değildir. Bu nedenle, sezgisel algoritmalarda kaotik harita kullanarak optimum noktalardan kaçmak ya da yerel optimuma takılma riskini azaltmak mümkün olabilmektedir. Bu çalışmada kaotik haritaların yeni optimizasyon yöntemlerinden olan Altın Sinüs Algoritması üzerindeki etkisi incelenmiştir. Anahtar kelimeler: Kaotik haritalar, kaos, Altın Sinüs Algoritması, sezgisel optimizasyon.

\section{Chaotic Gold Sine Algorithm for Numerical Optimization}

\begin{abstract}
Random number sequences are frequently used for stochastic structures of metaheuristic optimization algorithms. The distributions of randomly generated sequences of numbers are quite effective in the convergence of heuristic optimization algorithms to the global optimum. By gathering randomly generated numbers in a certain area or by generating the same values, the risk of the algorithm to be localized may be increased. Therefore, the numbers produced are not the same, the spread spectrum has the effect of the performance of heuristic algorithms. Chaotic maps are discrete-time systems, and the numbers produced by chaotic maps have a broad spectrum and are not periodic. Therefore, it is possible to avoid the optimum points by using the chaotic map in heuristic algorithms or to reduce the risk of local optimization. In this study, the effect of chaotic maps on the Golden Sine Algorithm, which is one of the new optimization methods, has been investigated.
\end{abstract}

Key words: Chaotic maps, chaos, Golden Sine Algorithm, metaheuristic optimization.

\section{Giriş}

Sezgisel yöntemlerin büyük bir kısmında rastgele sayı dizileri önemli bir yer tutmaktadır. Rastgele üretilen sayıların belli bir alanda toplanması veya aynı değerler üretilmesi ile algoritmanın lokal optimumlara takılma riski artabilmektedir. $\mathrm{Bu}$ nedenle üretilen sayıların aynı olmaması, yayılmış spektruma sahip olması sezgisel algoritmaların performansını etkileyen durumlardır.

Kaos teorisi, kaotik dinamik sistemlerin çalışmasını ifade eder. Kaotik sistemler, başlangıç koşullarına duyarlı doğrusal olmayan dinamik sistemlerdir. Başlangıç koşullarındaki küçük değişiklikler, sistemin sonucunda yüksek varyasyonlara neden olur. Kaotik sistemler rastgele davranışlar sergilese bile, bir sistemin kaotik davranışlar sergilemesi için mutlaka rastgele olması gerekmez. Başka bir deyişle, deterministik sistemler de kaotik davranışlar gösterebilir. Sezgisel algoritmalarda kaotik harita kullanarak optimum noktalardan kaçmak ya da yerel optimuma takılma riskini azaltmak mümkün olabilmektedir. Bu nedenle, optimizasyon algoritmalarının performanslarını arttırmak için rastgele sayı dizileri yerine kaotik haritalar yaygın şekilde kullanılmıştır. Bu çalışmalara örnek olarak doğadan esinlenmiş olan Kaotik Parçacık Sürü Optimizasyon Algoritması [1], Kaotik Arı Koloni Algoritması[2], Kaotik Genetik Algoritmalar [3], Kaotik Diferansiyel Gelişim [4], Kaotik Benzetilmiş Tavlama [5] ve Kaotik Ateşböceği Algoritması [6], Kaotik Kril Sürü Algoritmas1 [7], Kaotik Balina Optimizasyon Algoritmas1 [8] ve Kaotik Biyocoğrafya Tabanlı Optimizasyon [9] dur. Bu çalışmaların sonuçları, kaosun metasezgisel algoritmalara başarıyla uygulanabilirliğini teyit etmektedir. Bu çalışmanın amacı literatüre yeni kazandırılmış matematik tabanlı bir metasezgisel algortima olan Altın Sinüs Algortiması (ASA)[10]'nın kaotik versiyonları oluşturularak kaotik haritaların sezgisel algoritma üzerindeki etkisi incelenmiştir. ASA'nın seçilme sebebi ise kısıtsız kıyaslama fonksiyonlarında global optimumu bulmada diğer optimizerlerden daha başarılı sonuçlar vermesi ve daha az başlangıç parametresine sahip olmasıdır.

\footnotetext{
* Sorumlu yazar: etanyildizi@ firat.edu.tr. Yazarların ORCID Numarası: ${ }^{1}$ 0000-0003-2973-9389
} 


\section{Altın Sinüs Algoritması}

Sezgisel yöntemler deterministik yöntemlerden farklı olarak stokastik tabanlı operatörler kullanırlar. Bu operatörlerin oluşturulmasında farklı kaynaklardan (biyoloji, fizik, müzik, spor, matematik vb.) ilham alınmaktadır. Önerilen matematik tabanlı algoritmanın ilham kaynağı sinüs fonksiyonudur. Sinüs fonksiyonu değerlerini düzenli aralıklarla tekrar eden bir periyodik bir fonksiyondur. Sinüs fonksiyonun tüm değerlerinin birim çemberini taraması optimizasyon problemlerindeki arama uzayının taranmasına benzemektedir. $\mathrm{Bu}$ benzerlik ASA'nın geliştirilmesine ilham kaynağı olmuştur. ASA, sinüs fonksiyonun ve altın oran yöntemini birleştirerek aramayı geliştirmeyi amaçlamaktadır (Şekil 1) [10].

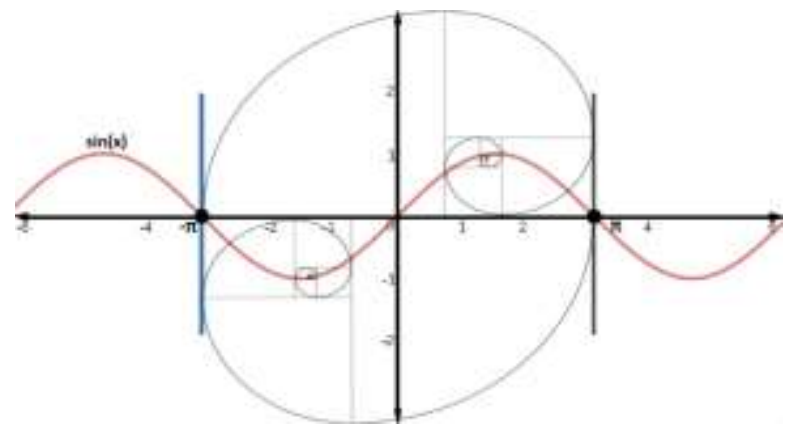

Şekil 1. Altın Sinüs Algoritması [10]

ASA'nın en iyi çözümü bulmak için Denklem 1'de verilen yapıyı kullanır.

$V(i, j)=V(i, j) \cdot\left|\sin \left(r_{1}\right)\right|-p \cdot \sin \left(r_{1}\right) \cdot \mid x_{1} \cdot D(j)-x_{2} \cdot V(i, j)$

Burada $V(i, j)$; $i$. çözümdeki $j$. boyutun mevcut değerini göstermektedir. $D$, belirlenen hedef değerdir. $r_{1}=[0,2 \pi]$ aralı̆ı̆ında rastgele bir sayıdır. $p=[0, \pi]$ aralığında rastgele bir sayıdır. $x_{1}$ ve $x_{2}$ ise altın kesit yöntemi ile elde edilen katsayılardır. Sezgisel yöntemlerin temel hedefi arama uzayının en iyi sonuç vereceği düşünülen alanlarını keşfetmek ve bu alanların da mümkün olduğunca tamamının taranmasını sağlamaktır. Arama uzayının geniş olması problemlerin çözümü için büyük bir sorun oluşturmaktadır. Arama uzayının daraltılması işleminin nasıl yapıldığı problemlerin çözümünde sonuçları önemli ölçüde etkilemektedir. ASA bu işlemi en iyi şekilde yapmak için alın kesit yöntemini kullanmaktadır. ASA'nın sözde kodu Şekil 2'de gösterilmektedir.

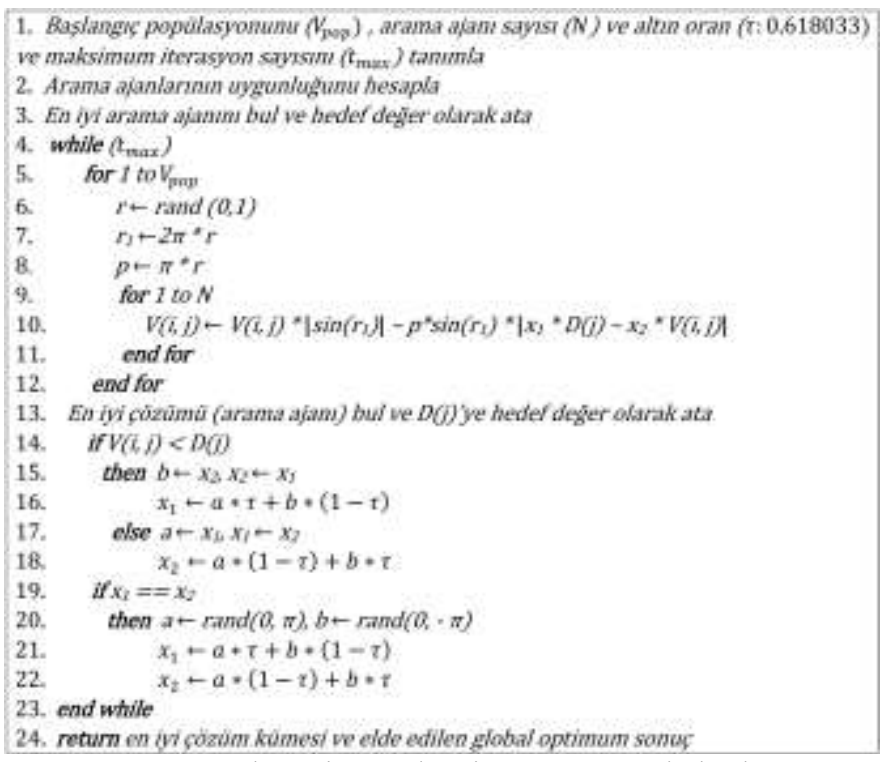

Şekil 2. Altın Sinüs Algoritmasının sözde kodu. 


\section{Kaotik Haritalı ASA Yöntemleri}

Literatürde birçok kaotik harita tanımlanmıştır. Bu çalışmada kullanılan kaotik haritalar Tablo 1 de verilmiştir. ASA'da rastgele değerler kullanan kısımlar kaotik harita denklemleri ile değiştirilerek ASA'nın on farklı kaotik versiyonu oluşturulmuştur.

Tablo 1. Kaotik Haritalar

\begin{tabular}{|c|c|c|c|}
\hline & Kaotik Harita & Foksiyon & \\
\hline K1 & Chebyshev & $x_{i+1}=\cos \left(i \cos ^{-1}\left(x_{i}\right)\right)$ & $(2)$ \\
\hline K2 & Çember & $x_{i+1}=\bmod \left(x_{i}+b-\left(\frac{a}{2 \pi}\right) \sin \left(2 \pi x_{i}\right), 1\right), a=0.5, b=0.2$ & (3) \\
\hline K3 & Gauss/Mouse & $x_{i+1}=\left\{\begin{array}{cc}1 & x_{i}=0 \\
\frac{1}{\bmod \left(x_{i}, 1\right)} & \text { aksi takdirde }\end{array}\right.$ & (4) \\
\hline K4 & İteratif & $x_{i+1}=\sin \left(\frac{a \pi}{x_{i}}\right) a=0.7$ & $(5)$ \\
\hline K5 & Lojistik & $x_{i+1}=a x_{i}\left(1-x_{i}\right), a=4$ & $(6)$ \\
\hline K6 & Parçalı & $x_{i+1}=\left\{\begin{array}{cc}\frac{x_{i}}{P} \\
\frac{x_{i}-P}{0.5-P} & 0 \leq x_{i}<P \\
\frac{1-P-x_{i}}{0.5-P} & P \leq x_{i}<0.5, \\
\frac{1-x_{i}}{P}\end{array}\right.$ & (7) \\
\hline K7 & Sinüs & $x_{i+1}=\frac{a}{4} \sin \left(\pi x_{i}\right) a=4$ & $(8)$ \\
\hline K8 & Singer & $x_{i+1}=\mu\left(7.86 x_{i}-23.31 x_{i}^{2}+28.75 x_{i}^{3}-13.302875 x_{i}^{4}\right), \mu=2.3$ & (9) \\
\hline K9 & Sinüzoidal & $x_{i+1}=a x_{i}^{2} \sin \left(\pi x_{i}\right), a=2.3$ & (10) \\
\hline K10 & Tent & $x_{i+1}=\left\{\begin{array}{c}\frac{x_{i}}{0.7} x_{i}<0.7 \\
\frac{10}{3}\left(1-x_{i}\right) x_{i} \geq 0.7\end{array}\right.$ & (11) \\
\hline
\end{tabular}

Altın Sinüs Algoritması'nda $p$ parametresi her arama ajanı için $[0, \pi]$ aralığında rastgele olarak belirlenmektedir. Rastgele üretilen sayılar bazen arama uzayının tamamının sömürülmesini engellemektedir. Seyedali Mirjalili ve Amir H. Gandomi [11], kaotik haritaları Denklem 12 ve Denklem 13 ile normalize ederek başarılı sonuçlar elde etmişlerdir. Bu çalışmada normalize edilmiş kaotik haritalara entegre olarak adaptif olarak azalan Denklem 1'de gösterilen $r_{1}$ deseni ilk kez kullanılarak arama uzayının mümkün olduğunca tamamının taranması hedeflenmektedir.

$V(t)=\operatorname{Max}-\frac{t}{T}(\operatorname{Max}-\operatorname{Min})$

Normalize $\left(K_{i}(t) ;[a, b]^{\prime}\right.$ dan $\left.[0, V(t)]^{\prime} y e\right): K_{i}^{n o r m}(t)=\frac{\left(K_{i}(t)-a\right) \times(V(t)-0)}{(b-a)}+0=\frac{\left(K_{i}(t)-a\right) \times(V(t))}{(b-a)}$

burada, $K$ : Kaotik harita, $i$ : Kaotik haritanın indeksi, $t$ : Mevcut iterasyon, $T$ : Maksimum iterasyon sayıs1, $[$ Max - Min]: Uyarlanabilir aralığı temsil eder. $[a, b]$ : Kaotik haritaların aralığını gösterir. $V(t)$ her iterasyonda azaltılırken her iterasyonda $[a, b],[0, V(t)]$ ile eşleşir. Bu, adaptif normalizasyon sürecini ifade eder. Sonuç olarak $p$ katsayısının değeri Denklem 13 kullanılarak güncelleştirilir.

$p(t)=K_{i}^{\text {norm }}(t)+c-t \times((c) / T)$

$p$ 'ye kaotik haritalar eklemek, hem adaptif $p$ 'nin hem de kaotik haritaların rastgele davranışına aynı anda katkıda bulunur. Kaotik haritalar, $p$ için farklı değerler sağlamaktadırlar. $p$ 'nin değerini aniden değiştirerek yerel minimumdan kurtulmaya yardımcı olarak, daha iyi yakınsama hızı sağlamaktadır. Uyarlamalı normalleştirme yaklaşımı ise kaos tabanlı ASA algoritmalarının arama evresinden sömürü evresine yavaş yavaş geçmesine yardımcı olur. 


\section{Deneysel Sonuçlar}

Global optimizasyon algoritmalarının performanslarını incelemek için farklı boyutlarda test fonksiyonlarının sayısal örneklerine göre değerlendirmek daha uygundur. Geliştirilen yeni yöntem literatürde yaygın olarak kullanılan beş farklı kıyaslama fonksiyonuna uygulanmıştır (Tablo 2) [12]. F1-F5, kalite testi fonksiyonlarına ait sonuçlar Tablo 3’te gösterilmektedir.

Tablo 2. Kalite test fonksiyonları

\begin{tabular}{|l|l|c|c|c|}
\hline & Foksiyon & Boyut & Aralık & Optimum \\
\hline F1 & Rosenbrock & 30 & {$[-5,10]$} & 0 \\
\hline F2 & Penalty(01) & 30 & {$[-50,50]$} & 0 \\
\hline F3 & Penalty(02) & 30 & {$[-50,50]$} & 0 \\
\hline F4 & Hartmann $(\mathrm{H} 3,4)$ & 6 & {$[0,1]$} & -3.86278 \\
\hline F5 & Hartmann $(\mathrm{H} 6,4)$ & 6 & {$[0,1]$} & -3.32237 \\
\hline
\end{tabular}

Tablo 3. Kalite testi fonksiyonları sonuçları ( $F$ :fonksiyon, $\dot{I}$ : istatistikler )

\begin{tabular}{|c|c|c|c|c|c|c|}
\hline$F$ & $\dot{I}$ & $\mathrm{~F} 1$ & F2 & F3 & $\mathrm{F} 4$ & F5 \\
\hline \multirow{4}{*}{ ASA } & Ortalama & $7.6412 \mathrm{e}-04$ & $1.5886 \mathrm{e}-06$ & $1.9802 \mathrm{e}-05$ & -3.7841 & -2.9592 \\
\hline & Std & 0.0011 & $3.0044 \mathrm{e}-06$ & $6.0729 \mathrm{e}-05$ & 0.0865 & 0.3052 \\
\hline & En iyi & $1.1344 \mathrm{e}-06$ & $1.0691 \mathrm{e}-08$ & $7.5857 \mathrm{e}-08$ & -3.8627 & -3.3036 \\
\hline & En kötü & 0.0042 & $1.3794 \mathrm{e}-05$ & $3.3367 \mathrm{e}-04$ & -3.5980 & -1.4908 \\
\hline \multirow{4}{*}{ K1-ASA } & Ortalama & $5.9770 \mathrm{e}-04$ & $1.8438 \mathrm{e}-06$ & $1.8587 \mathrm{e}-05$ & -3.7973 & -2.9281 \\
\hline & Std & 0.0017 & $4.6076 \mathrm{e}-06$ & $4.9544 \mathrm{e}-05$ & 0.0678 & 0.3884 \\
\hline & En iyi & $1.3246 \mathrm{e}-08$ & $4.5515 e-11$ & $2.4121 \mathrm{e}-09$ & -3.8624 & -3.3114 \\
\hline & En kötü & 0.0090 & $2.0640 \mathrm{e}-05$ & $2.1019 \mathrm{e}-04$ & -3.6667 & -1.6320 \\
\hline \multirow{4}{*}{ K2-ASA } & Ortalama & $7.1666 \mathrm{e}-04$ & $7.3447 \mathrm{e}-07$ & $1.1229 \mathrm{e}-05$ & -3.8036 & -2.9376 \\
\hline & Std & 0.0025 & $1.2626 \mathrm{e}-06$ & $2.5533 \mathrm{e}-05$ & 0.0766 & 0.2701 \\
\hline & En iyi & $6.9253 \mathrm{e}-08$ & $1.9028 \mathrm{e}-09$ & $2.6391 \mathrm{e}-09$ & -3.8626 & -3.2262 \\
\hline & En kötü & 0.0139 & $4.3577 \mathrm{e}-06$ & $1.2592 \mathrm{e}-04$ & -3.6089 & -1.7379 \\
\hline \multirow{4}{*}{ K3-ASA } & Ortalama & 0.0021 & $3.5520 \mathrm{e}-06$ & $2.3832 \mathrm{e}-05$ & -3.7973 & -2.8948 \\
\hline & Std & 0.0074 & $1.4880 \mathrm{e}-05$ & $4.5442 \mathrm{e}-05$ & 0.0745 & 0.4178 \\
\hline & En iyi & $5.6950 \mathrm{e}-08$ & $3.8111 \mathrm{e}-09$ & $3.5240 \mathrm{e}-09$ & -3.8622 & -3.2817 \\
\hline & En kötü & 0.0403 & $8.1773 \mathrm{e}-05$ & $1.9561 \mathrm{e}-04$ & -3.6044 & -1.1704 \\
\hline \multirow{4}{*}{ K4-ASA } & Ortalama & $8.4300 \mathrm{e}-04$ & $8.0683 \mathrm{e}-07$ & $5.7996 \mathrm{e}-05$ & -3.7849 & -2.9479 \\
\hline & Std & 0.0028 & $1.3490 \mathrm{e}-06$ & $1.7730 \mathrm{e}-04$ & 0.0818 & 0.3961 \\
\hline & En iyi & $4.6070 \mathrm{e}-08$ & $1.7830 \mathrm{e}-09$ & $1.0332 \mathrm{e}-08$ & -3.8627 & -3.2552 \\
\hline & En kötü & 0.0145 & $5.0969 \mathrm{e}-06$ & $9.5103 e-04$ & -3.6018 & -1.4650 \\
\hline \multirow{4}{*}{ K5-ASA } & Ortalama & 0.0023 & $1.6635 \mathrm{e}-06$ & $2.7116 \mathrm{e}-05$ & -3.7810 & -2.9439 \\
\hline & Std & 0.0072 & $4.6690 \mathrm{e}-06$ & $6.1372 \mathrm{e}-05$ & 0.0761 & 0.2922 \\
\hline & En iyi & $5.8983 \mathrm{e}-08$ & $4.6439 \mathrm{e}-10$ & $4.7801 \mathrm{e}-10$ & -3.8625 & -3.2723 \\
\hline & En kötü & 0.0306 & $2.4306 \mathrm{e}-05$ & $2.7017 \mathrm{e}-04$ & -3.6007 & -1.5972 \\
\hline \multirow{4}{*}{ K6-ASA } & Ortalama & $8.9644 \mathrm{e}-04$ & $1.7741 \mathrm{e}-06$ & $1.1126 \mathrm{e}-05$ & -3.8007 & -2.9447 \\
\hline & Std & 0.0014 & $5.0282 \mathrm{e}-06$ & $1.8043 \mathrm{e}-05$ & 0.0714 & 0.3346 \\
\hline & En iyi & $2.9675 \mathrm{e}-08$ & $2.5383 \mathrm{e}-10$ & $3.1385 \mathrm{e}-09$ & -3.8628 & -3.3052 \\
\hline & En kötü & 0.0052 & $2.6333 \mathrm{e}-05$ & $6.6479 \mathrm{e}-05$ & -3.5950 & -1.3301 \\
\hline \multirow{4}{*}{ K7-ASA } & Ortalama & $5.2884 \mathrm{e}-04$ & $1.2585 \mathrm{e}-06$ & $3.3608 \mathrm{e}-05$ & -3.7955 & -2.9658 \\
\hline & $S t d$ & 0.0010 & $3.6219 \mathrm{e}-06$ & $6.8010 \mathrm{e}-05$ & 0.0649 & 0.1243 \\
\hline & En iyi & $8.4680 \mathrm{e}-08$ & $4.3978 \mathrm{e}-10$ & $5.7179 \mathrm{e}-09$ & -3.8627 & -3.1448 \\
\hline & En kötü & 0.0052 & $1.5491 \mathrm{e}-05$ & $3.1941 \mathrm{e}-04$ & -3.6213 & -2.6929 \\
\hline \multirow{4}{*}{ K8-ASA } & Ortalama & $1.0084 \mathrm{e}-04$ & $7.2342 \mathrm{e}-07$ & $2.0493 e-05$ & -3.7863 & -2.9263 \\
\hline & Std & $1.7375 \mathrm{e}-04$ & $1.1624 \mathrm{e}-06$ & $3.6663 \mathrm{e}-05$ & 0.0795 & 0.3267 \\
\hline & En iyi & $4.3724 \mathrm{e}-08$ & $4.7235 \mathrm{e}-10$ & $4.6726 \mathrm{e}-09$ & -3.8614 & -3.2193 \\
\hline & En kötü & $8.3789 \mathrm{e}-04$ & $3.8443 \mathrm{e}-06$ & $1.2470 \mathrm{e}-04$ & -3.6099 & -1.3824 \\
\hline \multirow{4}{*}{ K9-ASA } & Ortalama & $2.7427 \mathrm{e}-04$ & $8.4349 \mathrm{e}-07$ & $1.7158 \mathrm{e}-05$ & -3.8101 & -2.9757 \\
\hline & Std & $9.3238 \mathrm{e}-04$ & $2.6673 e-06$ & $4.4625 \mathrm{e}-05$ & 0.0575 & 0.2004 \\
\hline & En iyi & $2.8276 \mathrm{e}-07$ & $4.3278 \mathrm{e}-10$ & $8.5489 \mathrm{e}-09$ & -3.8622 & -3.2979 \\
\hline & En kötü & 0.0051 & $1.4665 \mathrm{e}-05$ & $2.0350 \mathrm{e}-04$ & -3.6634 & -2.3267 \\
\hline \multirow{4}{*}{ K10-ASA } & Ortalama & 0.0015 & $1.7372 \mathrm{e}-06$ & $1.7069 \mathrm{e}-05$ & -3.7870 & -2.8510 \\
\hline & Std & 0.0042 & $3.6962 \mathrm{e}-06$ & $3.7362 \mathrm{e}-05$ & 0.0823 & 0.4274 \\
\hline & En iyi & $2.2575 e-09$ & $3.0208 \mathrm{e}-09$ & $4.6515 \mathrm{e}-08$ & -3.8623 & -3.1764 \\
\hline & En kötü & 0.0199 & $1.3951 \mathrm{e}-05$ & $1.3582 \mathrm{e}-04$ & -3.6016 & -1.1698 \\
\hline
\end{tabular}


Tablo 3'deki test sonuçlarından F1 fonksiyonunun çözümünde K10-ASA algoritmasının, F2 fonksiyonunun çözümünde K1-ASA algoritmasının, F3 fonksiyonunun çözümünde K5-ASA algoritmasının, F4 ve F5 fonksiyonlarının çözümünde K6-ASA algoritmasının optimum noktaya en yakın sonuçlar verdikleri görülmektedir. Kaotik haritalı ASA algoritmasının uygulandığı F1-F5 fonksiyonlarına ait ortalama yakınsama eğrileri Şekil 3’te verilmiştir.

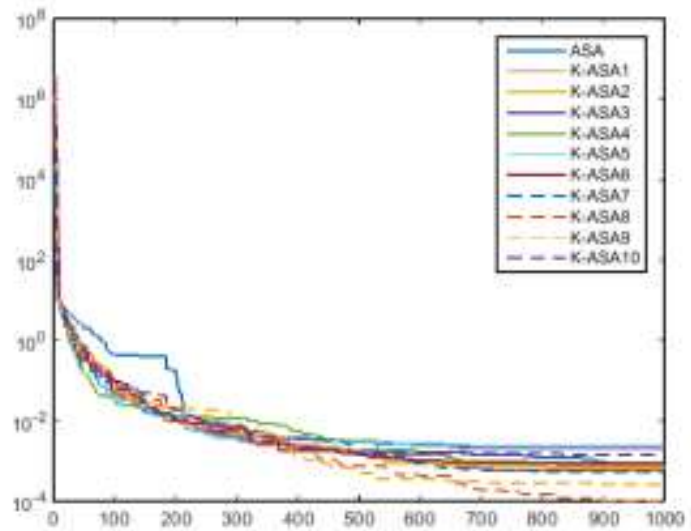

(a)

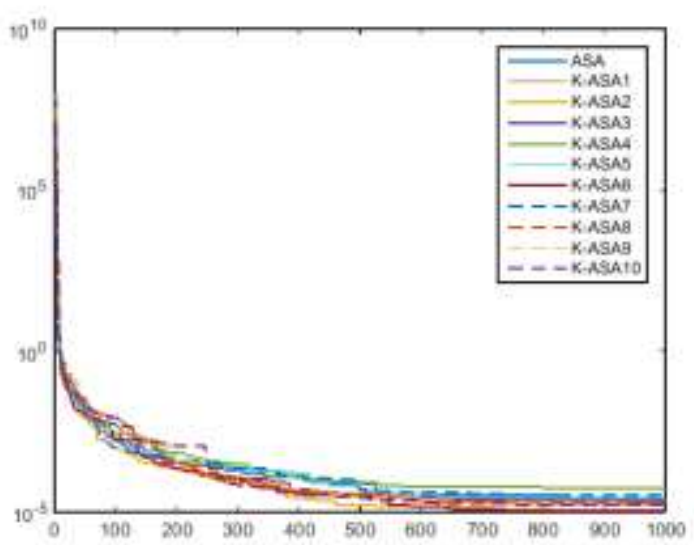

(c)

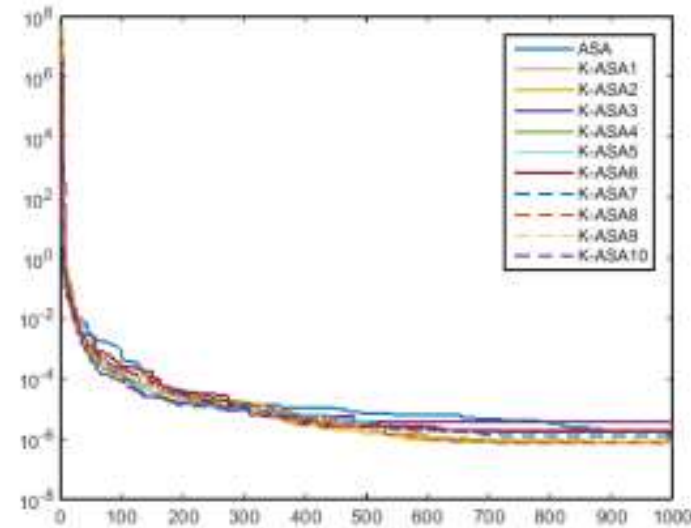

(b)

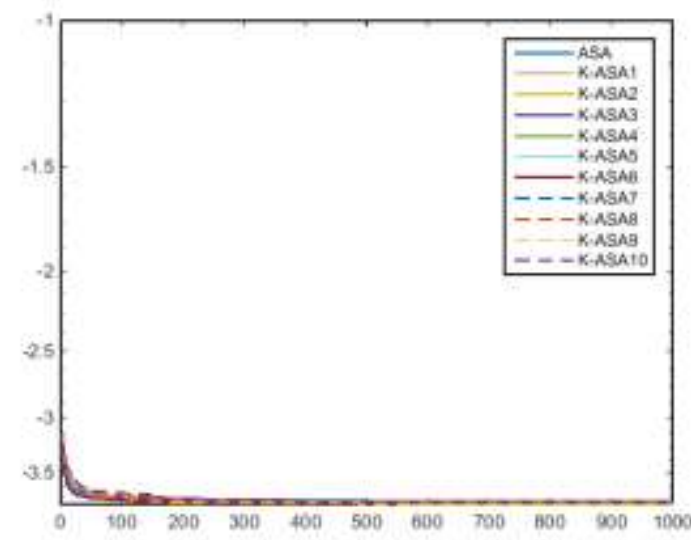

(d)

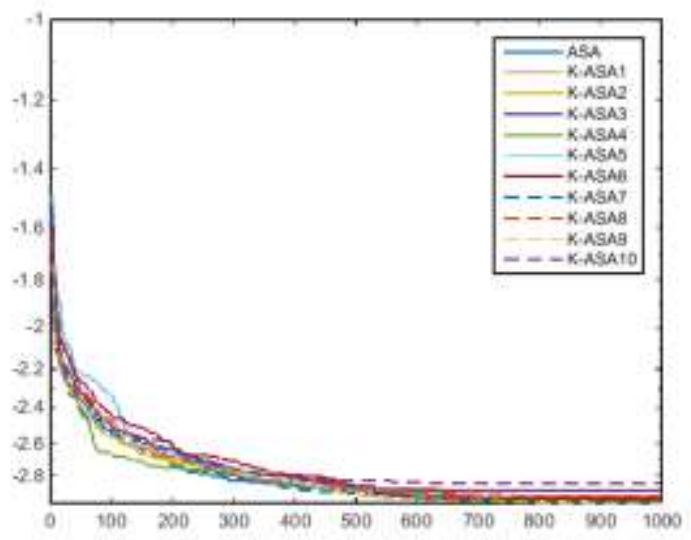

(e)

Şekil 3. Ortalama yakınsama eğrileri; a) F1 fonksiyonu, b) F2 fonksiyonu, c) F3 fonksiyonu, d) F4 fonksiyonu, e) F5 fonksiyonu 
Ortalama uygunluk eğrilerine bakıldığında F1 ve F2 fonksiyonu için K8-ASA algoritmasının iyi optimum değere sahip olduğu (Şekil 3a- Şekil 3b), F3 fonksiyonu için K8-ASA algoritmasının iyi optimum değere sahip olduğu (Şekil 3c), F4 ve F5 fonksiyonu için ise K9-ASA algoritmasının iyi optimum değere sahip olduğu (Şekil 3dŞekil 3e) görülmektedir. Temel algoritma ile kaotik algoritmalar arasındaki farkların istatistiki anlamlılık düzeyini gözlemlemek amacıyla Wilcoxon işaretli sıralar testi uygulanmıştır. Bu test her bir kaotik versiyon için en iyi algoritma ile temel algoritma çiftleri arasında uygulanmıştır (Tablo 4). Tablo 4'te $p$-değeri anlamlılık düzeyini $R^{+}$kaotik algoritmaların ve $R^{-}$ise temel algoritmanın rank toplamlarını göstermektedir.

Tablo 4. Wilcoxon işaretli sıralar testi karşılaştırma sonuçları

\begin{tabular}{|c|c|c|c|c|c|c|}
\hline $\mathrm{F}$ & $\dot{\mathrm{I}}$ & F5 & F12 & F13 & F19 & F20 \\
\hline \multirow{3}{*}{ ASA /K1-ASA } & $p$-değeri & 0.1915 & 0.5999 & 0.6583 & 0.5038 & 0.7343 \\
\hline & $\mathrm{R}^{+}$ & 169 & 207 & 211 & 200 & 216 \\
\hline & $\mathrm{R}^{-}$ & 296 & 258 & 254 & 265 & 249 \\
\hline \multirow{3}{*}{ ASA /K2-ASA } & $p$-değeri & 0.0285 & 0.1109 & 0.1986 & 0.2802 & 0.5999 \\
\hline & $\mathrm{R}^{+}$ & 126 & 155 & 170 & 180 & 258 \\
\hline & $\mathrm{R}^{-}$ & 339 & 310 & 295 & 285 & 207 \\
\hline \multirow{3}{*}{ ASA /K3-ASA } & $p$-değeri & 0.4653 & 0.2989 & 0.8774 & 0.5857 & 0.4779 \\
\hline & $\mathrm{R}^{+}$ & 197 & 182 & 240 & 206 & 267 \\
\hline & $\mathrm{R}^{-}$ & 268 & 283 & 225 & 259 & 198 \\
\hline \multirow{3}{*}{ ASA /K4-ASA } & $p$-değeri & 0.0428 & 0.2289 & 0.1986 & 0.8612 & 0.6583 \\
\hline & $\mathrm{R}^{+}$ & 134 & 174 & 295 & 241 & 211 \\
\hline & $\mathrm{R}^{-}$ & 331 & 291 & 170 & 224 & 254 \\
\hline \multirow{3}{*}{ ASA /K5-ASA } & $p$-değeri & 0.6143 & 0.1650 & 0.7189 & 0.7499 & 0.5577 \\
\hline & $\mathrm{R}^{+}$ & 208 & 165 & 250 & 248 & 261 \\
\hline & $\mathrm{R}^{-}$ & 257 & 300 & 215 & 217 & 204 \\
\hline \multirow{3}{*}{ ASA /K6-ASA } & $p$-değeri & 0.9918 & 0.1064 & 0.4284 & 0.6143 & 0.7655 \\
\hline & $\mathrm{R}^{+}$ & 233 & 154 & 194 & 208 & 247 \\
\hline & $\mathrm{R}^{-}$ & 232 & 311 & 271 & 257 & 218 \\
\hline \multirow{3}{*}{ ASA /K7-ASA } & $p$-değeri & 0.5038 & 0.1306 & 0.5440 & 0.9918 & 0.6143 \\
\hline & $\mathrm{R}^{+}$ & 200 & 159 & 262 & 233 & 257 \\
\hline & $\mathrm{R}^{-}$ & 265 & 306 & 203 & 232 & 208 \\
\hline \multirow{3}{*}{ ASA /K8-ASA } & $p$-değeri & 0.0068 & 0.0428 & 0.7971 & 0.9426 & 0.4165 \\
\hline & $\mathrm{R}^{+}$ & 101 & 134 & 245 & 236 & 272 \\
\hline & $\mathrm{R}^{-}$ & 364 & 331 & 220 & 229 & 193 \\
\hline \multirow{3}{*}{ ASA /K9-ASA } & $p$-değeri & 0.0148 & 0.0196 & 0.3709 & 0.0519 & 0.7971 \\
\hline & $\mathrm{R}^{+}$ & 114 & 119 & 189 & 138 & 220 \\
\hline & $\mathrm{R}^{-}$ & 351 & 346 & 276 & 327 & 245 \\
\hline \multirow{3}{*}{ ASA /K10-ASA } & $p$-değeri & 0.3493 & 0.4284 & 0.4779 & 0.7499 & 0.1306 \\
\hline & $\mathrm{R}^{+}$ & 187 & 194 & 198 & 248 & 306 \\
\hline & $\mathrm{R}^{-}$ & 278 & 271 & 267 & 217 & 159 \\
\hline
\end{tabular}

Wilcoxon işaretli sıralar testi karşılaştırma sonuçlarına göre Tablo 4 incelendiğinde algoritmalar arasında p>0.05 olduğundan anlamlı bir fark görülmemiştir. Ancak rank değerlerine bakıldığında K1-ASA, ASA'ya 5/0, K2-ASA, ASA'ya 4/1, K3-ASA, ASA'ya 3/2, K4-ASA ASA'ya 3/2, K6-ASA, ASA'ya 3/2, K7-ASA, ASA'ya 3/2, K9-ASA, ASA'ya 5/0, K10-ASA, ASA'ya 3/2 üstünlük sağlamıştır. ASA ise, K5-ASA'e 3/2, K8-ASA'e $3 / 2$ üstünlük sağlamıştır.

\section{Sonuçlar}

$\mathrm{Bu}$ çalışmada ASA'nın performansını iyileştirmek için on kaotik harita kullanılmıştır. Kalite testi fonksiyonları üzerinde testler yapılarak sonuçlar Wilcoxon işaretli sıralar testi ile desteklenmiştir. Sonuçlar, kaotik haritaların ASA'nın hem arama hem de sömürme evrelerini iyileştirme kabiliyetine sahip olduğunu kanıtlamaktadır. Ayrıca sonuçlar, Chebyshev ve Sinüzoidal kaotik haritanın en iyi olduğunu göstermektedir.

Kaotik haritaların ASA'nın, keşif aşamasını güçlendirir ve yerel minimuma sıkışmaktan kurtulmaya yardımcı olur. Önerilen yeni yaklaşımla keşif ve sömürünün adaptif olarak dengelenmesine izin verir. Başka bir 
deyişle, K-ASA'nın keşif evresinden sömürü evresine aşamalı olarak geçmesine yardımcı olur. Gelecek çalışmalarda, gerçek dünya mühendislik problemlerinin çözümü için K-ASA algoritmalarının kullanılması amaçlanmaktadır. Ayrıca, diğer kaotik haritaların da ASA'ya uygulanması hedeflenmektedir.

\section{Kaynaklar}

[1] Alatas B, Akin E, Ozer AB. Chaos embedded particle swarm optimization algorithms. Chaos, Solitons \& Fractals 2009. 40(4):1715-1734.

[2] Alatas B. Chaotic bee colony algorithms for global numerical optimization. Expert Syst Appl 2010. 37(8): 5682-5687.

[3] Jun-feng Y, Chi M, Xiao-qi P, Zhi-kun H, Jun H. A new optimization approach-chaosgenetic algorithm, Syst Eng 2001. $1: 1-5$.

[4] Zhenyu G, Bo C, Min Y, Binggang C. Self-adaptive chaos differential evolution. Adv Nat Comput 2006. 972-975.

[5] Mingjun J, Huanwen T. Application of chaos in simulated annealing. Chaos Solitons Fractals 2004. 21: 933-941.

[6] Gandomi AH, Yang XS, Talatahari S, Alavi AH. Firefly algorithm with chaos. Commun Nonlinear Sci 2012; 18(1):8998

[7] Wang GG, Guo L, Gandomi AH, Hao GS, Wang H. Chaotic krill herd algorithm. Inform Sciences 2014; 274:17-34

[8] Tanyıldızı E, Cigal T. Kaotik Haritalı Balina Optimizasyon Algoritması. Fırat Üniversitesi Mühendislik Bilimleri Dergisi 2017; 29(1):309-319.

[9] Saremi S, Mirjalili S, Lewis A. Biogeography-based optimisation with chaos. Neural Comput Appl 2014; 25:10771097.

[10] Tanyıldızı E, Demir G. Golden Sine Algorithm: A Novel Math-Inspired Algorithm. Adv Electr Comput En 2017; 17: $71-78$

[11] Mirjalili S, Gandomi AH. Chaotic gravitational constants for the gravitational search algorithm. Appl Soft Comput 2017. 53:407-419.

[12] Yao X, Liu Y, Lin G. Evolutionary programming made faster. IEEE T Evolut Comput 1999; 3(2): 82-102. 